$\begin{gathered}\text { Revista ELectrónica de } \\ \text { Investigación y EValuación } \\ \text { Educativa }\end{gathered}$
ISSN: 1134-4032

\title{
La evaluación de la producción científica: breve análisis crítico
}

\author{
Evaluation of scientific production: short critical analysis
}

\section{Giménez-Toledo, Elea}

Consejo Superior de Investigaciones Científicas (CSIC)

\begin{abstract}
Resumen
Se realiza una revisión histórica (centrada fundamentalmente en España, pero con múltiples referencias a otros ámbitos internacionales) del proceso de evaluación de las producción científica, especialmente de las publicaciones periódicas. Se analizan también las alternativas disponibles en la actualidad, tras una etapa convulsa que ha visto desaparecer diversos instrumentos para la evaluación de revistas. Por último se identifican algunas tendencias y necesidades en el ámbito de la evaluación de publicaciones científicas que presumiblemente marcaran el desarrollo futuro de futuros instrumentos y procedimientos.
\end{abstract}

\section{Palabras clave:}

Evaluación; Comunicación Científica; Publicación Científica Revistas; Revistas Científicas
Fecha de recepción 30 de marzo de 2015

Fecha de aprobación 21 de abril de 2015

Fecha de publicación 21 de abril de 2015

\begin{abstract}
A historic review (fundamentally centered in Spain, but with multiple references to other international fields) of the evaluation process of scientific production has been carried out, mainly of periodicals. Available alternatives have also been analyzed after a turbulent period that has seen varied instruments for journal revision disappear. Finally, some of the tendencies and necessities in the field of scientific publication evaluation that will presumably mark the future development of future instruments and procedures are identified.
\end{abstract}

Keywords:

Evaluation Scientific; Communication; Scientific Publishing Journals; Scientific Journals
Reception Date

2015 March 30

Approval Date

2015 April 21

Publication Date:

2015 April 21
Todo aniversario lleva asociado un necesario balance. La conmemoración de los veinte años de la revista RELIEVE representa una extraordinaria ocasión para echar la vista atrás y revisar críticamente el escenario de la evaluación científica, que tanto afecta a la edición de revistas académicas. La larga andadura de RELIEVE le ha permitido asistir a todos los cambios que se han producido en el ámbito académico, desde la intensificación de las evaluaciones científicas a la aparición de la ANECA, desde la edición científica más o menos amateur a una más profesionalizada, desde el acceso restringido al acceso abierto a los contenidos científicos, desde la existencia de una única fuente de indicadores bibliométricos a la generación de fuentes con indicadores para revistas científicas nacionales.

Las publicaciones científicas constituyen el núcleo fundamental de la evaluación de la actividad investigadora. No es un dato nuevo pero sí necesario para contextualizar el objeto de este artículo: cómo se aborda la evaluación de las publicaciones, qué indicadores se eligen, quienes los producen y cómo afecta todo ello a investigadores, evaluadores y editores. 


\section{Modelo de evaluación en las agencias de evaluación españolas}

La creación de la CNEAI (1989), el nacimiento de ANECA (2002) y la inminente fusión de ambas ${ }^{[1]}$, prevista para 2015, han marcado en cierto modo el comportamiento y la evolución de las revistas científicas españolas, especialmente las dedicadas al ámbito de las Humanidades y Ciencias Sociales. El establecimiento de criterios para la evaluación de la producción científica -pilar fundamental de la evaluación- de profesores e investigadores se puede traducir, en la práctica, como el establecimiento de directrices de edición para revistas científicas. Solo aquellas que cumplen los parámetros establecidos por CNEAI y ANECA tienen posibilidades de ser bien evaluadas $\mathrm{y}$, por tanto, serán las más buscadas por los autores españoles. Al fin y al cabo del "canal" que elijan (revista, editorial del libro, etc.) depende gran parte de su éxito en la evaluación a la que se sometan.

A su vez, los criterios definidos por estos organismos de evaluación han estado basados en algunas cuestiones entre las que han debido encontrarse la propia política científica del país y las prioridades que establece, las prácticas internacionales de evaluación y los trabajos desarrollados por distintos grupos de investigación españoles. Sobre este último punto, hay evidencias clarísimas. Los sistemas de información e indicadores sobre revistas científicas españolas mencionados explícitamente tanto por CNEAI como por ANECA fueron creados por dos grupos de investigación: el grupo EC3 de la Universidad de Granada y el Grupo EPUC ${ }^{[2]}$ del CSIC. También los criterios que forman parte del sello de calidad FECYT y aquellos que definen a las revistas científicas que CNEAI considera entre las "evaluables" proceden de los indicadores definidos por ambos grupos de investigación.

Resulta importante destacar este hecho por varias razones: los criterios de evaluación se apoyan en evidencias que resultan de la investigación; las agencias demuestran su permeabilidad a los resultados de investigación generados por grupos especializados y, por tanto, se tiene en cuenta el criterio científico/técnico, además del político; la transferencia efectiva de la investigación realizada desde los grupos a las agencias. No siempre la formulación o la aplicación de los criterios es todo lo correcta o precisa que pudiera ser y eso a veces genera falta de aceptación por parte de los investigadores evaluados. Sin embargo, es importante reconocer que en la mayor parte de los casos los criterios utilizados por las agencias de evaluación no son improvisados y que están propuestos o apoyados por los investigadores que componen las comisiones temáticas.

La revisión de las distintas resoluciones de CNEAI, publicadas periódicamente en el BOE a lo largo de los años, permite observar hasta qué punto han ido evolucionando los criterios de evaluación, tanto en transparencia y precisión como en adaptación a los hábitos de comunicación científica de los distintos campos. Desde el inicial criterio selectivo publicar o no en revistas de la Web of Sciencea los criterios diferenciados por áreas y que consideran otras fuentes de información, ha habido una clara evolución. No quiere decir esto que los modelos de evaluación sean perfectos ni tampoco que las distintas comunidades científicas acepten plenamente los criterios sino, más bien, que en los comités temáticos de las agencias de evaluación se interacciona directa o indirectamente tanto con los investigadores (a través de asociaciones científicas, por ejemplo) como con los grupos de investigación especializados en evaluación científica. Esa interacción permite ir evolucionando en los criterios de evaluación y, de alguna manera, responder mejor a la realidad de las prácticas de comunicación científica de los investigadores.

\section{Características básicas del sistema actual de evaluación de la producción científica}

ANECA y CNEAI, las dos agencias de evaluación científica de ámbito estatal que realizan evaluación de investigadores (a nivel 
individual), entre otras, comparten algunas características muy relevantes en el modo de evaluar las publicaciones científicas. Analizando los dos documentos de referencia que marcan los criterios de evaluación, pueden identificarse algunos rasgos característicos de ambas que, de paso, sirven de recordatorio para evitar los siempre frecuentes malentendidos en la evaluación científica. De una forma esquemática, podría decirse que ambas:

- Siguen un proceso de "informed peer review”, mediante el cual la producción científica de los investigadores es evaluada a partir del juicio de los expertos que componen cada panel temático y de indicadores bibliométricos que sirven como referencia o apoyo.

- Tienen definidos distintos criterios de evaluación en función del ámbito disciplinar que están evaluando $\mathrm{y}$, por ejemplo, los criterios empleados para evaluar la Biomedicina no son los mismos que los que se utilizan para evaluar la Filología. Los distintos comités temáticos están integrados por especialistas en las distintas disciplinas que abarcan y tienen voz sobre los criterios que se aplican ${ }^{[3]}$, lo que significa que estos se adecúan, al menos en parte, a las características de la comunicación científica en esas disciplinas.

- Se evalúa el canal, no el artículo, el capítulo o los libros concretos. Aunque existe la posibilidad de analizar en cierto modo el contenido (a ello contribuyen la "defensa" que hacen de los artículos sus autores, en el marco de los procesos de evaluación de CNEAI, por ejemplo)

- En términos generales, tienen más peso los artículos de revista que los libros o capítulos de libros. En la mayor parte de disciplinas, se espera que los investigadores generen artículos como resultados de su investigación. Solo en algunas disciplinas de Humanidades y Ciencias Sociales se prevé como resultado la producción de libros o capítulos de libros. Los textos de referencia sobre el procedimiento de evaluación de las agencias permiten deducir la equivalencia entre el peso de un artículo y el peso de un libro: un libro equivaldría a dos artículos o a un artículo y un capítulo de libro.

- En la evaluación de los libros se tienen en cuenta distintos indicadores de calidad, pero apenas hay fuentes que recojan valores para esos indicadores.

- En la evaluación de revistas, la Web of Science y sus Journal Citation Reports son las fuentes preferentes de referencia en los procesos de evaluación. La ausencia o presencia de una revista en las bases de datos que integran la WoS, así como el factor de impacto reflejado en los JCR, son indicadores clave $-\mathrm{y}$ predominantes sobre el resto- en los procesos de evaluación en España.

- Cuando una publicación no tiene indicadores en WoS/JCR, entonces se consideran otras fuentes, nacionales e internacionales. Para las Humanidades y las Ciencias Sociales, destacan en el ámbito internacional ERIH y Scopus. ERIH está en pleno proceso de cambio, tal y como se comentará más adelante, mientras que Scopus sigue aumentando el número de revistas indexadas, siendo así menos selectiva que WoS y generando unos indicadores (a través de Scimago Journal Rank), aún no considerados expresamente por las agencias de evaluación. En relación con las fuentes que aportan indicadores para revistas españolas, si bien se contemplan y se citan varias de forma expresa, lo cierto es que muchas de ellas han dejado de estar operativas y actualizadas, por falta de financiación. También este asunto será tratado más adelante.

- Aunque la ley de la Ciencia representa una clara apuesta por el acceso abierto, la evaluación científica está basada en fuentes "cerradas". En los datos que ofrece Delgado (2015) sólo un 10,6\% de las revistas recogidas en JCR son de acceso 
abierto. Las revistas mejor valoradas (según JCR) no son OA o son híbridas. Esto supone que el investigador ha de optar:

- por el acceso abierto pleno, es decir, eligiendo una revista en abierto que no cobre por publicar. De este modo, garantiza la libre disponibilidad de sus resultados de investigación, tal y como promueve la Ley de la Ciencia; sin embargo, esta acción tendrá repercusiones negativas en su evaluación.

- por el pago por publicación en abierto, detrayendo de los fondos para investigación recursos que van a parar a grandes grupos editoriales.

- por publicar en una revista cerrada (a la que se accede por suscripción), normalmente perteneciente a grandes grupos editoriales y recogidas en JCR, lo que facilita una evaluación positiva.

\section{Una revisión crítica de las fuentes sobre revistas}

Poco hay que decir ya sobre la principal fuente de información empleada para evaluación (WoS). Son innumerables los estudios basados en la producción científica, las citas y el impacto a partir de WoS. También lo son aquellos que muestran las limitaciones del producto para la evaluación de algunas áreas y las precauciones que deben tomarse. Asimismo, son sobradamente conocidas las características de Scopus. Aunque es una fuente secundaria atendiendo a la jerarquía de fuentes que establecen las agencias de evaluación en España, su mayor cobertura de revistas y los indicadores que proporciona directa o indirectamente, la convierten en una fuente habitual en los estudios bibliométricos.

No cabe duda de que la internacionalidad y la multidisciplinariedad de ambas las hacen idóneas para hacer análisis de productividad e impacto de todo tipo. Tampoco cabe duda del interés que tienen para los gobiernos, pues les permiten obtener fácilmente indicadores que son comparables entre países y, por tanto, tener estándares y objetivos a los que apuntar. La otra ventaja destacable es que, al estar producidas por grandes corporaciones, el acceso a los datos solo depende del pago de la suscripción correspondiente. El alto precio que tienen las suscripciones a ambos recursos se debe ver compensado por las ventajas apuntadas.

Sin embargo, aunque este punto de vista resulta comprensible también lo es el hecho de que no representan bien la totalidad de la producción científica de calidad que se genera en las distintas disciplinas y en los distintos países. Por esa razón, muchos países del mundo y algunas "regiones" (Europa con ERIH o América Latina con Latindex) se plantearon y se plantean la creación de sistemas alternativos, nacionales o supranacionales de indicadores para la producción científica generada en cada país, al margen de la recogida en WoS o Scopus. Estas iniciativas se pueden agrupar en los siguientes modelos:

- Desarrollo de CRIS (Current Research Information Systems) a nivel nacional que, además, llevan aparejados ciertos indicadores de calidad para la producción científica recogida. Es el caso del sistema belga o del noruego (Sivertsen, 2010)

- Categorizaciones de revistas que se aplican a la producción científica que se somete a evaluación. Fue el caso de la ANEP en España, es el caso de la ANVUR en Italia, de AERES en Francia o de Publindex en Colombia por poner solo algunos ejemplos. También ERIH podría incluirse en este apartado, aunque teniendo en cuenta las reservas que siempre han tenido sus creadores para que tal categorización se aplicara a evaluaciones de individuos.

- Sistemas de evaluación de revistas científicas, aglutinadores de distintos de calidad indirectos; entre ellos se pueden encontrar el de ámbito regional, Latindex, que engloba a revistas de América Latina, el Caribe, España y 
Portugal y los de ámbito nacional RESH y DICE

- Sistemas de evaluación que muestra una selección de revistas que cumplen con los criterios definidos, aunque no tiene la función de mostrar el valor de indicadores (el cumplimiento del mismo) para cada revista. Es el caso de los denominados "núcleos básicos" de revistas $^{[4]}$ creados en algunos países latinoamericanos o incluso podrían considerarse aquí las revistas fuente incluidas en Scielo o en Redalyc.

- Índices de impacto nacionales: Polish Sociology Citation Index, Russian Citation Index o los españoles In-RECS, $\underline{\text { In- RECJ e In-RECH. }}$.

Basten estos ejemplos para mostrar que la evaluación de revistas científicas nacionales no es una ocurrencia coyuntural de un país en concreto sino que responde a una clara necesidad por contar con indicadores que informen sobre el sector editorial de las revistas de un país, que ayuden a tomar decisiones en evaluación científica informando de aquello que no está cubierto por WoS y que, además, sirva para otros fines como fijar ciertos estándares de calidad o promover las mejoras en la edición. Sin sistemas de evaluación nacionales la evaluación resultante será sesgada y, por otra parte, el conocimiento del sector editorial será deficiente.

En América Latina fueron pioneros en la definición de criterios de calidad para las publicaciones científicas y los sistemas de evaluación nacionales que tienen se han ido desarrollando de desigual manera en función de los momentos y de los países. En España, el trabajo sobre esta cuestión se empezó a consolidar a finales de los 90. Entre 2004 y 2006 ya se habían hecho públicas las primeras versiones de RESH, DICE e In-RECS. Empezarían entonces a proliferar los estudios sobre calidad de las revistas, a surgir otros sistemas, como el aún vigente MIAR y a crearse iniciativas desde el ámbito más político/institucional, como el sello de calidad
FECYT. Es importante destacar los orígenes, pues en aquellas fechas eran pocos los países europeos que contaban con sus propias herramientas de evaluación, aunque empezaban ya a gestarse.

Conociendo esta evolución, no deja de resultar paradójico que una década más tarde ninguno de los sistemas pioneros en España y en Europa (RESH, DICE e In-RECS) estén ya operativos y que la razón no sea científica sino económica: falta de financiación para mantenerlos. Mientras, en distintos países europeos ya han creado sus propios sistemas que gozan de apoyos institucionales importantes y que garantizan, en cierto modo, su viabilidad. Este hecho ofrece una pauta interesante de cara al futuro. Si bien los grupos de investigación tienen capacidades y competencias para desarrollar los sistemas de evaluación de publicaciones, son las instituciones, y especialmente aquellas que tienen responsabilidades en el ámbito de la política científica, quienes deben promover, sustentar y respaldar los sistemas. Al fin y al cabo la información que ofrecen estos sistemas son un servicio público, sirven a muchos agentes del ámbito académico y, por otra parte, cumplen una función que es la de "proteger" el patrimonio científico que suponen las revistas o, dicho de otro modo, dar la oportunidad a las buenas revistas científicas, no indexadas en Wos, de ser bien evaluadas. No hay que olvidar que a través de las universidades se destinan muchos fondos económicos a la edición de revistas que, paradójicamente, son minusvaloradas en algunos procesos de evaluación en los que prima la WoS. Se produce aquí cierta incoherencia entre los esfuerzos realizados para editar, mediante la inversión de recursos públicos, y el reconocimiento a la hora de evaluar; esto afecta especialmente a las revistas altamente especializadas (Mañana-Rodríguez, 2013), tanto geográfica como temáticamente.

En defensa de aquellos sistemas hay que decir que fueron sistemas considerados como fuente de referencia en las agencias de evaluación españolas; de hecho, aún lo son, a 
pesar de que sus informaciones están desactualizadas. Ese hecho permitió, por primera vez, evaluar un conjunto de la producción científica mucho mayor que el que está incluido en la Web of Science, cuestión que resulta fundamental en Humanidades y Ciencias Sociales. Sirva como contundente ejemplo el informe de la CRUE (Michavila, 2012). Según los datos que ofrece -referidos a la producción científica generada por la comunidad universitaria y obtenidos en 2010, la publicación de artículos supuso en Arte y Humanidades un $44 \%$ del total de la producción científica del área (7763 artículos). Entre ellos, solo un $15 \%$ son artículos cubiertos por la WoS. Eso significa que de no utilizarse otras fuentes de evaluación complementarias a la WoS se está desestimando para evaluación (o evaluando sin herramientas que garanticen cierta objetividad) un $85 \%$ de los artículos producidos en estas disciplinas. En algunas ocasiones ha sido la propia comunidad universitaria la que ha criticado la existencia de estas herramientas. Quizá este dato cuantitativo relativo a la parte de sus obras que permanecen invisibles o infravaloradas sin estas herramientas de evaluación sea suficientemente concluyente.

A esto hay que añadir que los sistemas de evaluación de revistas no sirven solo a efectos de evaluación individual o institucional. Sirven para tener un conocimiento más global del sector editorial de un país, para conocer mejor el comportamiento de las disciplinas, para identificar el rigor en la edición y/o la profesionalidad, etc. Todas ellas son cuestiones que podrían o deberían interesar a quienes están poniendo recursos en la edición de revistas; a los propios editores, que pueden "verse" en relación a otros editores y que, además, tienen un marco de referencia de los estándares internacionales de edición científica, un horizonte al que tender; a los autores que tendrán un mejor conocimiento de las revistas de su área; y, por supuesto, a los gestores de política científica que, además de contar con herramientas para la evaluación científica, podrían diseñar programas de apoyo a las publicaciones.

El caso es que, en España, estos sistemas han desaparecido (o no tienen la utilidad que tenían) y que, en cierto modo, se produce un retroceso. Aunque los documentos de referencia de las agencias siguen mostrándolas como herramientas complementarias en la evaluación, lo cierto es que su desactualización hace que la evaluación de la producción científica vuelva a estar basada casi exclusivamente en la WoS y también en Scopus.

Cabe decir que ni el esfuerzo ni el tiempo se perdieron en esos proyectos. En la edición científica de revistas se ha dado un salto cualitativo importante. En primer lugar, porque se edita mejor de lo que se editaba; desde el punto de vista formal, la mayor parte de las revistas siguen las directrices internacionales de edición científica, algo que no sucedía hace quince o veinte años, entre otras cosas porque se desconocían; desde el punto de vista de la calidad de los contenidos también se ha avanzado pues muchas de las revistas han mejorado sustancialmente sus políticas editoriales y sus filtros. No cabe duda de que hay una mayor y mejor conocimiento de lo que implica la buena edición científica. Otra cosa diferente es que todas las revistas que se publican sigan los mejores caminos o velen por la máxima calidad de los artículos. De la misma manera que algunos editores han visto en el establecimiento de indicadores y en la evaluación científica una oportunidad para revisar y replantearse sus proyectos editoriales, avanzando hacia una mayor calidad de los contenidos y hacia la profesionalización de la edición, otros han optado solo por aparentar el cumplimiento de los indicadores pero sin modificar la práctica editorial; esta opción es visible tanto para quienes trabajan con indicadores como para los evaluadores. 


\section{Herramientas vigentes, complementarias a WoS y Scopus}

Quedan aún dos sistemas que, siendo exigentes de una manera comparable a WoS, podrían llenar el vacío que dejan los sistemas de evaluación de revistas nacionales. Uno de ellos es el europeo ERIH. El European Reference Index for the Humanities que auspiciara y desarrollara la European Science Foundation es ya hoy un producto transferido a la Norwegian Social Sciences Data Services. Ha cambiado de productores y también de denominación ERIH Plus es ahora un sistema que incluye revistas de Humanidades pero también de Ciencias Sociales. En el momento de redactar este artículo la información que se ofrece para cada revista es, prácticamente, la editorial $y$, en algunos casos, se da la indicación de si es una publicación "peer reviewed”. Claro que el sistema está en transición pues se están evaluando las nuevas solicitudes de revistas para entrar en el sistema $\mathrm{y}$, por otra parte, cabe esperar que ofrezcan alguna información cualitativa sobre las publicaciones o bien que el propio proceso de selección sea tan riguroso que la sola presencia de la revista en ERIH Plus sea un signo inequívoco de calidad. La ausencia o presencia de una revista en ERIH Plus sería la única manera de utilizar, a día de hoy, el índice a efectos de evaluación. Sin embargo, a pesar de que el sistema puede ser uno de los referentes claros para la evaluación de la producción en Humanidades y Ciencias Sociales, está en fase embrionaria y necesita aún consolidarse.

El otro sistema es CIRC, una categorización de revistas que resulta de la agregación de información contenida en distintas fuentes de información sobre revistas. Es algo así como el resumen de lo que otras bases de datos dicen de una revista. Fue el resultado de una colaboración científica entre varios grupos de investigación españoles (Torres-Salinas et al., 2010) aunque, posteriormente, ha pasado a ser un desarrollo de la empresa EC3 metrics. En su origen integraba fuentes nacionales e internacionales, lo que la convertía en una fuente eficiente y sólida para evaluar la producción científica de un departamento, de un instituto de investigación, etc. En la actualidad, sus productores han anunciado la publicación de una nueva versión de manera inminente. Los cambios previsibles que están por venir son claros: al no haber fuentes nacionales de indicadores, CIRC deberá estar más basado en las fuentes internacionales existentes lo que, sin quitarle interés a la herramienta, representa de nuevo un retroceso, pues se excluye de la evaluación a un gran número de revistas españolas.

Existe otra fuente de datos cada vez más utilizada, aunque no oficialmente que es Google Scholar. No tiene el carácter "cerrado" del resto de fuentes pues constantemente actualiza sus datos e informaciones y no selecciona revistas, de tal forma que aporta información para todas. A partir de Google Scholar se pueden obtener datos de revistas y rankings para las mismas, tal y como vienen haciendo el Statistical Cybermetrics Research Group de la Universidad de Wolverhampton, el grupo EC3 de la Universidad de Granada, o la aplicación Publish or Perish (Harzing, 2007). Sin duda, es una fuente adicional para la evaluación científica que representa una gran ventaja: la producción de la "base de datos” depende de una gran empresa y, por tanto, se trata de explotar sus datos. Por otro lado, también tiene inconvenientes relacionados con el valor o la representatividad de las citas en las Humanidades y en las Ciencias Sociales y la posibilidad de manipular la información contenida en Google Scholar (Delgado et al., 2014).

En cualquier caso, la facilidad con que se obtienen los indicadores de impacto y su disponibilidad hacen que su uso se consolide en algunos países.

\section{Conclusiones}

Analizando la evaluación de revistas científicas que se ha dado en España desde que las agencias de evaluación intensificaron su actividad -en el caso de CNEAI- o se crearon -en el caso de ANECA, se pueden sacar algunas conclusiones. 
En primer lugar, aunque sea una obviedad decirlo, parece claro que la Web of Science, $y$ sus JCR, van a persistir como fuentes de referencia en la evaluación. Al haberse convertido en fuente internacional de referencia, su uso permite la comparabilidad de los procesos de evaluación y de las exigencias a investigadores; es un estándar que muchos países están dispuestos a compartir. Si a ello se une la tradición o inercia en su uso, el asentamiento de la herramienta, la posibilidad de modular las exigencias por áreas en función de los cuartiles, el liderazgo de Thomson Reuters y su influencia en las políticas de evaluación, parece imposible vislumbrar un escenario en el que la WoS no sea la fuente de referencia clave en los procesos de evaluación. Al menos en países que, como España, sigan los procesos de evaluación basados en el denominado "informed peer review", es decir, aquellos que combinan la utilización de indicadores bibliométricos con el juicio más cualitativo de los paneles de expertos. Existe el modelo del Reino Unido, que en los criterios definidos para su Research Excellence Framework (REF), excluye expresamente la utilización de indicadores bibliométricos, datos sobre citas o categorizaciones de revistas para la evaluación de la actividad científica que realizan. El sistema de evaluación es cualitativo: está basado en el juicio de los especialistas del área. Humanistas y científicos sociales han venido reivindicando modelos de evaluación cualitativos frente a los cuantitativos y parece que un sistema como el británico podría gozar de mayor aceptación. Pero también es necesario indicar que estos sistemas son muy costosos y que, si acaso, podrán permitírselos aquellos países con una intensa actividad e inversión en investigación.

El camino intermedio lo constituyen, sin duda, las categorizaciones o sistemas de evaluación de revistas que permiten atender a distintas características de las publicaciones cualitativas y cuantitativas- al tiempo que suponen una oportunidad de conocer el sector editorial en su conjunto y, en consecuencia, los déficits que pueda tener y las acciones que puedan llevarse a cabo para defender la buena edición científica, que no tiene cabida en bases de datos internacionales.

\section{Referencias}

Cetto, A. M., Alonso-Gamboa, J. O., Córdoba González, S., Giménez-Toledo, E. \& Chávez Sánchez, G. (2012). Organized access to the IberoAmerican quality journals: The PPL experience. Scholarly and Research Communication, 4 (1), p.19.

Delgado López-Cózar, Emilio (2015). Las revistas electrónicas en acceso abierto: pasado, presente y futuro. RELIEVE, 21(1), art. M1. DOI: $\underline{10.7203 / \text { relieve.21.1.5005 }}$

Delgado López-Cózar, E., Robinson-García, N., \& Torres-Salinas, D. (2014). The Google Scholar Experiment: how to index false papers and manipulate bibliometric indicators. Journal of the Association for Information Science and Technology, 65(3), 446-454.

Giménez-Toledo, E., Román-Román, A., \& AlcainPartearroyo, M. D. (2007). From experimentation to coordination in the evaluation of Spanish scientific journals in the humanities and social sciences. Research evaluation, 16(2), 137-148.

Harzing, A.W. (2007). Publish or Perish. Disponible en http://www.harzing.com/pop.htm

Ley 15/2014, de 16 de septiembre, de racionalización del Sector Público y otras medidas de reforma administrativa. Disponible en https://www.boe.es/boe/dias/2014/09/17/pdfs/BO E-A-2014-9467.pdf

Mañana Rodríguez, J. (2013). Análisis multidimensional de la especialización en publicaciones de Ciencias Sociales $y$ Humanidades. Tesis doctoral. Disponible en http://e-archivo.uc3m.es/handle/10016/16964

Michavila, Francisco (dir.). (2012). La Universidad española en cifras. Madrid: CRUE. http://www.crue.org/Publicaciones/Documents/U EC/LA_UNIVERSIDAD_ESPANOLA_EN_CIF RAS.pdf

Sivertsen, G. (2010). A performance indicator based on complete data for the scientific publication output at research institutions. ISSI Newsletter 6, 22-28

Torres-Salinas, D., Bordons, M., Giménez-Toledo, E., Delgado-López-Cózar, E., Jiménez-Contreras, E., \& Sanz-Casado, E. (2010). Clasificación integrada de revistas científicas (CIRC): propuesta 
de categorización de las revistas en ciencias sociales y humanas. El profesional de la información, 19(6), 675-684.

Winclawska, B. M. (1996). Polish sociology citation index (principles for creation and the first results). Scientometrics, 35(3), 387-391.

\section{Notas}

[1] Ley 15/2014, de 16 de septiembre, de racionalización del Sector Público y otras medidas de reforma administrativa https://www.boe.es/boe/dias/2014/09/17/pdfs/B OE-A-2014-9467.pdf (Artículos 8 y 9)
${ }^{\text {[2] Hoy transformado en Grupo de Investigación }}$ sobre el Libro Académico (ÍLIA): http://ilia.cchs.csic.es

${ }^{[3]}$ El Director de Política Científica afirmaba en la Asamblea de la UNE de noviembre de 2014, que las comisiones de la CNEAI son soberanas, luego tienen capacidad de decisión en el modo en que se evalúa.

${ }^{[4]}$ Véase el caso de Argentina http://www.caicytconicet.gov.ar/nucleo-basico-de-revistascientificas/

\section{Autor}

To know more / Saber más

Giménez-Toledo, Elea (elea.gimenez@cchs.csic.es).

Científica titular del CSIC, es licenciada y doctora en Documentación.

Dirige el Grupo de Investigación sobre el Libro Académico (ÍLIA). Su actividad investigadora se centra en la actualidad en el libro académico y en los procesos de evaluación de la actividad científica en ciencias humanas y sociales. Ha sido coautora de las plataformas de evaluación de revistas DICE, RESH y CIRC y responsable de la parte española de Latindex. Es creadora del ranking de editoriales SPI (Scholarly Publisher Indicators). Su dirección postal es: Centro de Ciencias Humanas y Sociales (CCHS). Consejo Superior de Investigaciones Científicas (CSIC). Albasanz, 26-28. 28037 Madrid.

\section{RELIEVE}

\section{Revista ELectrónica de Investigación y EValuación Educativa E-Journal of Educational Research, Assessment and Evaluation}

[ISSN: 1134-4032]

(C) Copyright, RELIEVE. Reproduction and distribution of this articles it is authorized if the content is no modified and their origin is indicated (RELIEVE Journal, volume, number and electronic address of the document).

(C) Copyright, RELIEVE. Se autoriza la reproducción y distribución de este artículo siempre que no se modifique el contenido y se indique su origen (RELIEVE, volumen, número y dirección electrónica del documento). 